\title{
论 文 有机分析研究进展专刊
}

\section{天然产物的质谱智能解析研究}

\author{
夏兵, 马风伟, 丁立生, 周燕* \\ 中国科学院成都生物研究所, 成都 610041 \\ *通讯作者, E-mail: zhouyan@cib.ac.cn
}

收稿日期: 2017-07-13; 接受日期: 2017-09-15; 网络版发表日期: 2017-11-22

国家自然科学基金(编号: 21572221,21572219,21672206,21302180,21072185)和中国科学院昆明植物研究所植物化学与西部植物资源持续 利用国家重点实验室开放课题(编号：P2015-KF09)资助项目

\begin{abstract}
摘要串联质谱在鉴定天然产物的分子结构方面起着重要的作用。每一类天然产物, 在串联质谱中除有 常规的质谱裂解方式外, 通常还具有因特定骨架类型而产生的特征裂解. 因此可以通过天然产物各类成分 的特征质谱裂解所产生的特征碎片离子(diagnostic ions)来确认该类天然产物的结构类型, 然后再由常规的 裂解方式来推测其周边可能的取代基团. 基于以上思路, 我们设计并构建了一种天然产物质谱智能解析软 件 SmartMass.
\end{abstract}

关键词 天然产物, 质谱, 特征裂解, 质谱智能解析

\section{1 引言}

质谱(mass spectrometry, MS)作为一种能够获知化 合物分子质量信息的有力工具, 以其快速、灵敏、准 确等优点,在生物、化学、医药等领域发挥着越来越 重要的作用 ${ }^{[1,2]}$. 天然产物是植物、动物和微生物的次 生代谢产物(secondary metabolite), 结构复杂多样, 常 见的类型有黄酮、生物碱、皇苷、萜类、葱醌、木 脂素等. 这些成分具有不同的生物活性, 是天然药物 发挥作用的活性物质基础, 也是创新药物发现的重要 来源 ${ }^{[3 \sim 5]}$. 自 1994年以来, 美国食品药品管理局(FDA) 所批准的新药中, 源于天然产物的药物约占了一半 ${ }^{[6,7]}$. 长期以来, 由于天然产物结构的复杂性和多样性, 其 结构的解析工作常遇到很多困难. 传统的天然产物化 学成分研究一般需要通过提取、分离和纯化, 获得单
体化合物后才能够进行结构鉴定 ${ }^{[8,9]}$. 然而这种耗时费 力的研究方法常常只能获得含量较高、结构稳定的 成分. 自然界中大量存在的微量或痕量成分, 在分离 纯化过程中容易丢失, 最终难以获得单体化合物进行 结构鉴定 ${ }^{[10,11]}$.

核磁共振波谱与质谱是天然产物结构鉴定的主 要工具. 然而由于核磁共振波谱检测限和灵敏度的局 限性, 使其在微量天然产物成分鉴定中面临挑战. 随 着液相色谱-质谱联用技术、高分辨质谱和多级质谱 裂解技术的发展 ${ }^{[12,13]}$, 特别是多级质谱裂解技术在天 然产物快速篮查中的广泛应用, 逐渐成为了微量和不 稳定天然产物成分快速解析的有力手段 ${ }^{[14,15]}$. 最近几 年, 许多新的质谱技术大量出现, 使得质谱在分析微 量样品, 如细胞内的药物代谢物成为可能 ${ }^{[16,17]}$. 此外, 在代谢组学研究当中, 质谱技术是目前最主要的分析

引用格式: 夏兵, 马风伟, 丁立生, 周燕. 天然产物的质谱智能解析研究. 中国科学: 化学, 2017, 47: 1458-1466

Xia B, Ma F, Ding L, Zhou Y. Intelligent interpretation of mass spectra of natural products. Sci Sin Chim, 2017, 47: 1458-1466, doi: 10.1360/N03201700115 
手段之一, 广泛应用于代谢组学各个领域 ${ }^{[18]}$.

\section{2 天然产物的质谱智能解析}

质谱和串联质谱在化合物结构的鉴定和表征方面 已经成为了一个重要的信息来源. 现在已有利用质谱 信息辅助化合物结构鉴定的开发软件. 作为第一个利 用质谱信息解析化合物结构的程序, DENDRAL ${ }^{[19]}$ 首开 结构解析软件中使用质谱信息的先河, 并且启发了很 多后来软件程序如MOLGEN ${ }^{[20,21]}$ 等. 此外, 科学家们 还开发了一系列利用质谱信息的软件系统, 如Mass Frontier ${ }^{[22]} 、 A C D / M S$ Fragmenter ${ }^{[23]}$ 和 MASCOT $^{[24]}$ 等. Mass Frontier和 ACD/MS Fragmenter是目前比较领先 的商业软件, 他们搜集了一些质谱裂解方面较常见的 和启发式推导出的规则, 并利用这些规则来对化合物 的串联质谱裂解碎片进行预测, 且将预测到的结果与 实验数据相比较以确证候选化合物结构. Mass Frontier 针对化合物的 $\mathrm{n} 、 \pi$ 及 $\sigma$ 离子化反应并结合常见的裂解 规则及从文献中收集到的规则来预测化合物的裂解 碎片. ACD/MS Fragmenter则利用了类似的常见裂解 规则. 这几种常见的质谱信息软件特点对比见表 1 .

基于我们前期对天然产物不同类别的化合物特 征质谱裂解研究的基础上, 开发了质谱智能解析软件 SmartMass $^{[25]}$,一个基于上述理念的具有跨平台性和可 扩展性的天然产物结构解析程序. 与前面提到的其他 软件不同的是, SmartMass 主要致力于从串联质谱数 据中发现特征裂解模式的一定骨架类型的化合物, 尤 其是天然产物的结构解析.

\subsection{SmartMass 的基本工作流程}

虽然天然产物结构的复杂性和多样性极为丰
富, 通常可以将其划分为不同的类型, 如萜类、生 物碱、黄酮等, 但许多天然产物的结构具有共同的分 子骨架. 他们之间结构的区别主要是由周边取代基 不同导致的，这些化合物可以看成是属于同一亚类. 对这样的化合物进行串联质谱分析, 通常会出现一 些特征的裂解模式，这些模式可以反过来推测化合 物的结构类型. 因此, 基于质谱裂解规则的天然产物 结构解析可以简单地总结为: (1) 结构/骨架类型确 定; (2) 可能的周边基团扫描; (3) 其他手段的候选化 合物确证.

图1所示为SmartMass的基本工作流程示意. 待解 析未知化合物的串联质谱数据以纯文本形式 $(m / z$ 值和 对应的离子丰度使用空格或制表符分隔)输入软件, 并 通过处理去噪、排序并转换成Python列表, 这个列表 再加上母离子的 $m / z$ 值及加合离子的精确质量数一起 输入 SmartMass分析. 解析过程分为以下 5 个步骤:

1. 在裂解模式数据库中检索对应的解析规则, 如 果未知化合物MS/MS 谱图匹配上某一解析规则的特 征诊断模式的话即返回该解析规则.

2. 对该未知化合物的MS/MS应用步骤1返回的解 析规则以便计算出化合物的可能周边基团, 然后将解 析出来的周边基团嫁接回结构框架上. 在无解析规则 返回的情况下扫描化合物的MS/MS 谱图以获得该化 合物可能含有的周边基团或亚结构.

3. 结合前面 $1 、 2$ 步返回的信息, 从化合物数据库 如MyMolDB ${ }^{[26]} 、$ ChemSpider和PubChem ${ }^{[27,28]}$ 下载候选 化合物.

4. 利用化合物结构类型及其他可用的信息对下 载下来的候选结构进行篮选.

5. 进行其他用户自定义的后处理.

表 1 几种常见的质谱信息软件的特点

\begin{tabular}{|c|c|c|c|}
\hline 软件名称 & 工作原理 & 优点 & 缺点 \\
\hline DENDRAL & $\begin{array}{c}\text { 利用质谱数据与结构的经验规则, } \\
\text { 机器学习算法等进行推测 }\end{array}$ & 启发式计算, 预定义规则较少 & $\begin{array}{c}\text { 可能输出较多不可能存在的 } \\
\text { 结构, 准确性较低 }\end{array}$ \\
\hline MOLGEN & $\begin{array}{l}\text { 利用元素组成产生大量的分子结 } \\
\text { 构, 并通过虚拟裂解质谱与实验 } \\
\text { 质谱数据的匹配度进行过滤 }\end{array}$ & 较高结构生成效率 & $\begin{array}{c}\text { 产生的化合物结构数量庞大, 正确 } \\
\text { 结构可能被淹没而难以确定 }\end{array}$ \\
\hline Mass Frontier & $\begin{array}{l}\text { 收集整理了大量文献中的质谱裂解 } \\
\text { 规律, 结合虚拟裂解匹配和质谱谱库 }\end{array}$ & $\begin{array}{c}\text { 准确率较高, 功能强大, 结合自家 } \\
\text { 的质谱仪器平台, 操作简便 }\end{array}$ & 价格昂贵 \\
\hline ACD/MS Fragmenter & $\begin{array}{c}\text { 收集整理了常见质谱裂解规律对 } \\
\text { 质谱谱图进行碎片结构预测 }\end{array}$ & $\begin{array}{c}\text { 准确率较高, 功能强大, 结合自家 } \\
\text { 的质谱仪器平台, 操作简便 }\end{array}$ & 价格昂贵 \\
\hline
\end{tabular}




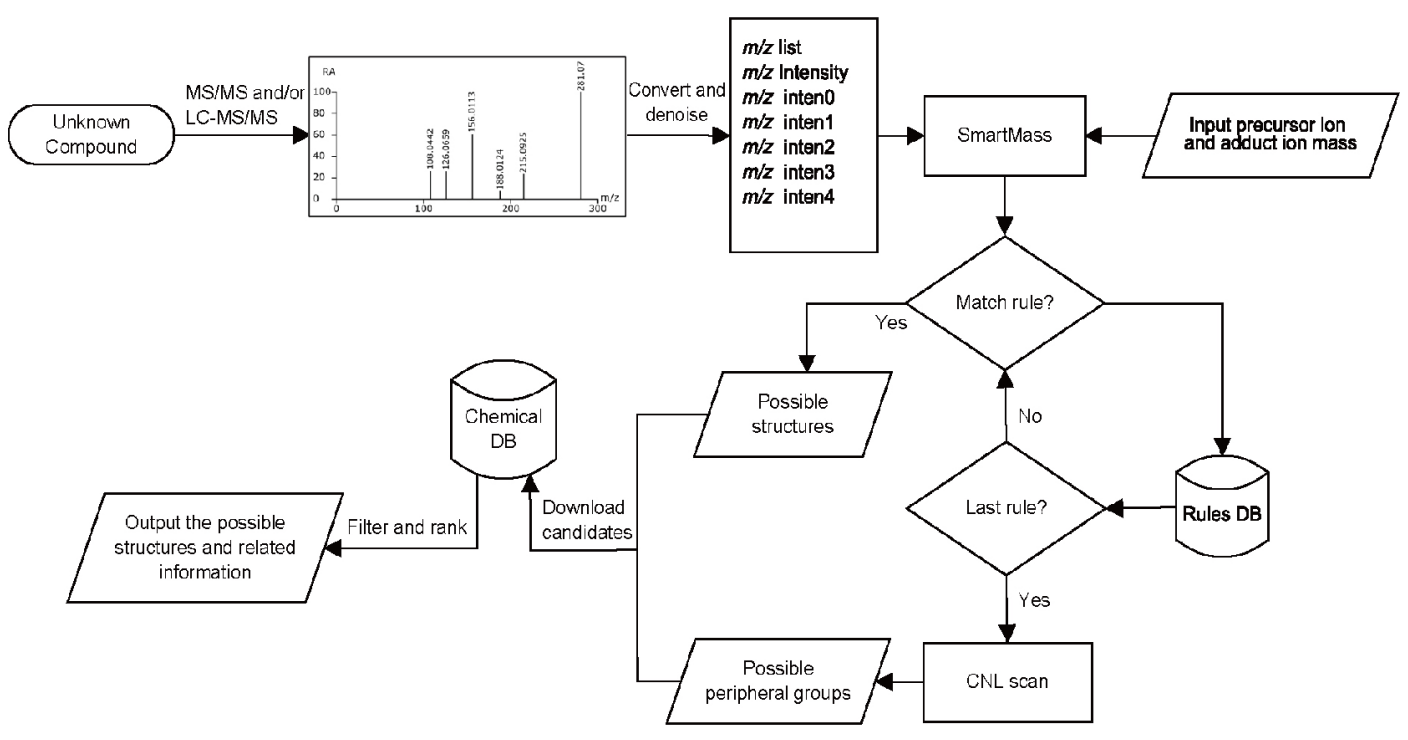

图 1 SmartMass 的架构及主要工作流程

\section{2 解析规则}

在所输入的MS/MS数据上执行解析代码是本软 件的核心工作. 解析代码本质上是一段特化的Python 代码, 此代码存储于一个SQLite数据库中的pattern表 中. 每一个解析代码都为解析某一种特定结构类型的 骨架而设. 此骨架之上有许多可能发生变化的基团. 它们均被编上号, 以便代码在处理的时候能够计算出 对应编号基团的可能结构. 为了方便编写解析代码, 系统预设了许多类和函数, 如 MS 类、Generator类和 Exact_mass函数等. MS类(该类实例代表了当前正在处 理的MS/MS谱图)提供了许多可能会被使用到的方法 如exists(*peaks), 该方法在所给出的峰出现在 MS/MS 谱图中的时候返回真; inten(peak)则返回一给定 $m / z$ 值 在谱图中对应的丰度; exist_nls $\left({ }^{*} \operatorname{loss}\right)$ 则当谱图中存在 给定中性丢失的时候返回真. Generator类则被用来动 态可控地产生周边基团的结构, 如 Generator.chain(n, element)返回一个 $\mathrm{n}$ 元的由 element组成的长链骨架. Exact_mass (formula, charge) 函数接受一个天然产物各 类成分的质谱裂解规律给定的分子式和电荷量作为 参数, 并且返回对应的精确质量数. 由于SmartMass可 以处理高分辨谱图, 所以在计算精确质量数时, 考虑 了离子所带电荷所涉及电子的质量. 同样被提供的还 有一些预定义的变量, 如MA代表母离子的 $m / z$ 值, 而 $\mathrm{AI}$ 则代表加合离子的 $m / z$ 值, AI对正离子来说是正值
而对负离子来说则是负的. 所有上述预定义的类、函 数和变量在解析代码的任何地方均可以随便使用, 这 些预定义为用户编写自己的解析代码提供了方便.

解析代码由 3 部分组成: 预定义部分、特征诊断 模式部分和解析及返回部分. 在预定义部分, 可以书 写任何合理的Python代码,这些代码可以在其他部分 被使用. 特征诊断模式部分实际上是一个逻辑判断表 达式, 只有这部分代码的运算结果为真, 这个解析规 则才被认为是所解析的MS/MS对应的规则, 只有这种 情况下, 整个规则才会被应用到MS/MS数据上对其进 行解析. 解析及返回部分的代码则负责根据人们总结 的规则计算出周边基团的精确质量数或者动态生成 周边基团的结构并将这些结果返回出去. SmartMass 接下来处理返回的结果并且在周边基团数据库中查 询可能的周边基团, 然后将所查到的基团嫁接到规则 所对应的结构骨架上形成一个候选结构, 这些候选结 构则会利用Indigo的API自动计算出一个合理的2D坐 标, 然后被软件渲染出来供大家做进一步判断.

\section{3 结果笁选及确认}

上述步骤所获得的候选结构将被用来在远程或者 本地数据库中进行查询以获得可能存在的候选结构. 现 在有很多化合物数据库服务如PubChem和ChemSpider 等可以免费使用. 他们中的一些甚至还支持网络API, 因此可以被SmartMass所调用. SmartMass如今默认支 
持PubChem和MyMolDB的网络API, 更多的支持可以 使用SmartMass插件系统自行添加或者按照系统支持 的两种数据库例子进行修改编写. 本地数据库支持尚 未完成.

从远程数据库中下载得到的候选结构可以利用 解析代码对应的结构类型及其他可得信息如周边基 团及母离子的同位素分布等进行进一步篮选, 这一步 工作是利用Rdkit的API完成的.

\section{SmartMass的应用}

\section{1 多环多异戊烯基间苯三酚(PPAPs)化合物的 分析}

多环多异戊烯基间苯三酚 (polycyclic polyprenylated acylphloroglucinols, PPAPs) 类化合物是从藤黄科 (Guttiferae)植物如连柱金丝桃(Hypericum cohaerens)、 Clusia species和 Garcinia species等中提取出来的一种 天然产物, 具有广泛的生物学活性. PPAPs类化合物具 有天然产物中少见的桥环、螺环乃至金刚烷等复杂 的核心结构, 且往往带有多个异戊烯基取代基. 常见 的PPAPs化合物可以分为两类, 即类型 $\mathrm{A}$ 和 $\mathrm{B}$, 这两种 类型的骨架如图2所示 ${ }^{[29]}$.

我们对类型 $\mathrm{B}$ 的PPAPs类化合物做过质谱裂解研 究 ${ }^{[28]}$. 对 B类型PPAPs化合物在负离子模式下的串联 质谱研究发现, 这类化合物的二级质谱中都给出了特 征的 $\mathrm{m} / \mathrm{z} 177$ 离子, 该特征离子的结构见图3. 因此, 离 子 $m / z$ 177可以用作这里化合物的一个特征碎片. 由于 PPAPs化合物是被高度取代的, 所以, 仅仅想通过串联 质谱数据就解析出某一个此类化合物的所有未知基 团是不可能的. 因此, 我们的解析规则只是作为一个 化合物类型探测器, 当发现某一化合物的质谱类型为
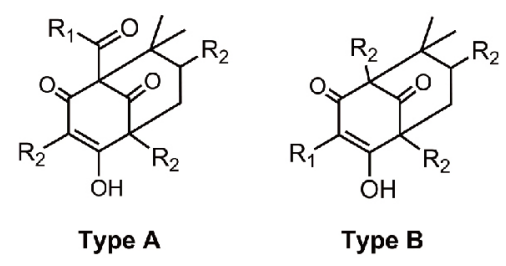

图 2 PPAPs化合物的两种常见结构类型

PPAPs时, 即给出其核心骨架类型, 然后再通过化合物
的准确分子量从网络的化合物数据库下载候选结构, 然后通过骨架类型篮选最终缩小候选化合物的范围, 为我们的进一步结构解析提供有益参考. 通过对 $\mathrm{B}$ 类 型PPAPs化合物的裂解规律进行总结, 提出了其裂解 途径.

以化合物 guttiferones $\mathrm{K}$ 为例, 其裂解规律如图3所 示.

通过对PPAPs化合物裂解规律的总结和特征诊断 离子的确定, 我们设计了对这类化合物的解析规则: (1) 检查未知化合物的 MS/MS谱图中是否存在 $m / z 177$ 峰, 如果存在, 则该化合物有可能是B类型的PPAPs化 合物; (2) 利用化合物的精确质量数从化合物数据库下 载候选结构; (3) 利用化合物特殊的骨架类型及常见 取代基(异戊烯基)对候选结构进行过滤(这一步不在 解析规则代码里面体现). 我们根据此解析策略编写 了解析规则代码, 其代码见S1 小节(网络版支持信息).

我们手动输入了某PPAPs化合物的MS/MS数据 到SmartMass里面, 在正确设置好了极性、加合离子 和母离子的 $m / z$ 值之后, 点击 Analyze按钮进行分析. SmartMass利用我们编写的解析规则, 正确地识别出 了该 MS/MS为一B类型PPAPs化合物的谱图, 并给出 了其骨架类型. 点击Confirm Results 以后, 程序便利用 精确质量数在PubChem数据库中进行自动检索和候选 结构下载得到1000多个候选结构, 利用PPAPs特征结 构进行过滤之后最终剩下了 33 个结构, 而正确的结构 正在其中 (guttiferones K). 上述分析过程见图S1 (网络 版支持信息).

\section{2 林可霉素类化合物的解析}

林可霉素 (lincosamides, 如 lincomycin 和 clindamycin等)是一类微生物代谢产物, 通过干扰细菌体 内蛋白质合成途径从而达到杀灭细菌目的的杀菌药 物. 林可霉素对格兰仕阳性菌具有很好的抗菌活性, 同时也可以用于杀灭弓形虫、衣原体、支原体和恶 性症原虫等. 本课题组之前已经完成了对林可霉素化 合物的 MS/MS裂解, 通过分析此类化合物的串联质谱 数据, 给出了其裂解途径(见图4).

林可霉素类化合物的 MS/MS谱图中具有特征的 $\left[\mathrm{C}_{6} \mathrm{H}_{12} \mathrm{~N}+n^{*} \mathrm{CH}_{2}\right]^{+}$及 $\left[\mathrm{MA}-\mathrm{HSCH}_{3}\right]^{+}$碎片 $(n$ 为林可霉素化 合物脂肪链的 $\mathrm{CH}_{2}$ 个数, $\mathrm{MA}$ 为母离子的 $m / z$ 值), 从图 5 中 可以看到, 常见的林可霉素化合物具有 3 个可能会不 


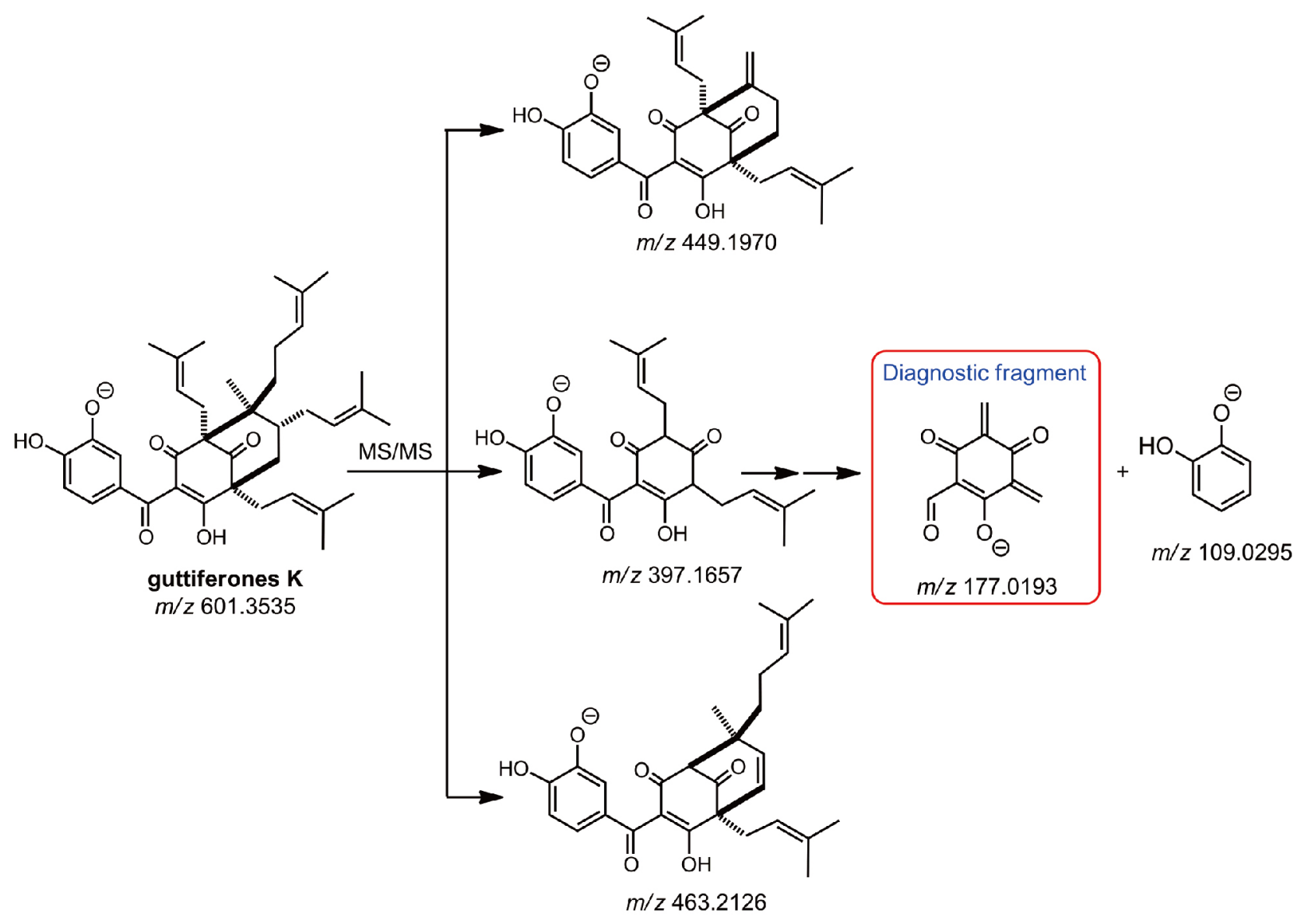

图 3 Guttiferones $\mathrm{K}$ 的串联质谱裂解途径及特征诊断离子的结构

一样的基团, 这些基团被标记为 $\mathrm{Ja}, \mathrm{Jb}$ 和 $\mathrm{Jc}$, 这些基团 的质量数可以利用在林可霉素串联质谱中出现的其 他碎片计算得到(计算公式见图4右侧). 其中脂肪链 $\mathrm{Jb}$ $\left(n^{*} \mathrm{CH}_{2}\right)$ 可以直接通过诊断离子 $\left[\mathrm{C}_{6} \mathrm{H}_{12} \mathrm{~N}+n^{*} \mathrm{CH}_{2}\right]^{+}$计算 得出, 即

$$
\mathrm{Jb}=\left[\mathrm{C}_{6} \mathrm{H}_{12} \mathrm{~N}+n * \mathrm{CH}_{2}\right]^{+}-\mathrm{C}_{6} \mathrm{H}_{12} \mathrm{~N}-\mathrm{ME}
$$

其中, $\mathrm{ME}$ 为电子的质量. 在林可霉素的串联质谱中会 出现直接掉 $\mathrm{HJa}$ 的峰, 及 $[\mathrm{MA}-\mathrm{HJa}+\mathrm{H}]^{+}$峰, 通过这个峰 的质荷比, 即可算出 $\mathrm{Ja}$ 的质量:

$$
\mathrm{Ja}=\mathrm{MA}-[\mathrm{MA}-\mathrm{HJa}]^{+}-\mathrm{H}
$$

同样, Jc的质量也可以计算得到:

$$
\mathrm{Jc}=\mathrm{MA}-\left[\mathrm{MA}-\mathrm{Jc}-\mathrm{C}_{3} \mathrm{H}_{5} \mathrm{OS}\right]^{+}-\mathrm{C}_{3} \mathrm{H}_{5} \mathrm{OS}
$$

从上面几个公式可以计算出所有 3 个未知基 才. 然而存在的一个问题是, 怎么在串联质谱里 面将这些峰, 即 $\left[\mathrm{C}_{6} \mathrm{H}_{12} \mathrm{~N}+n * \mathrm{CH}_{2}\right]^{+}$、 $[\mathrm{MA}-\mathrm{HJa}+\mathrm{H}]^{+}$和
$\left[\mathrm{MA}-\mathrm{Jc}-\mathrm{C}_{3} \mathrm{H}_{5} \mathrm{OS}\right]^{+}$识别出来.

事实上, $\left[\mathrm{MA}-\mathrm{Jc}_{\mathrm{c}}-\mathrm{C}_{3} \mathrm{H}_{5} \mathrm{OS}\right]^{+}$这个峰还可以表示为 $\left[\mathrm{C}_{13} \mathrm{H}_{24} \mathrm{~N}_{2} \mathrm{O}_{4}+\mathrm{Ja}+\mathrm{Jb}-\mathrm{ME}\right]^{+}$, 所以若知道 $\mathrm{Ja}$ 和 $\mathrm{Jb}$ 则可以计 算出 Jc. $n$ 是确定 $\mathrm{Jb}$ 的关键参数, 而 $n$ 的取值有一定范 围, $n$ 必然大于 1 , 小于 $\left[\left(\right.\right.$ 分子离子质荷比 $-\mathrm{C}_{6} \mathrm{H}_{12} \mathrm{~N}$ 的质量 数 $) / \mathrm{CH}_{2}$ 的质量数]. 因此, 只需将 $n$ 在此范围内变化, 然后 检查串联质谱里面是否出现 $\left[\mathrm{C}_{6} \mathrm{H}_{12} \mathrm{~N}+n^{*} \mathrm{CH}_{2}\right]^{+}$的峰即可. 如果出现, 则返回此时的 $n$ 值, 然后 $\mathrm{Jb}$ 的质量数也就确定 了. 对于 $\mathrm{Ja}$, 已经知道 $\mathrm{Ja}=\mathrm{MA}-[\mathrm{MA}-\mathrm{HJa}+\mathrm{H}]^{+}-\mathrm{H}+\mathrm{ME}$, 那 么对整个串联质谱的峰进行一个遍历, 遍历的值存储在 变量 $p 1$ 里面, 假设在某个值的时候, $p 1=[\mathrm{MA}-\mathrm{HJa}+\mathrm{H}]^{+}$, 则必然有以下关系:

$$
\mathrm{Jb}=n * \mathrm{CH}_{2}
$$

$$
\begin{aligned}
\mathrm{Ja} & =\mathrm{MA}-[\mathrm{MA}-\mathrm{HJa}+\mathrm{H}]^{+}-\mathrm{H} \\
& =\mathrm{MA}-p 1-\mathrm{H}
\end{aligned}
$$




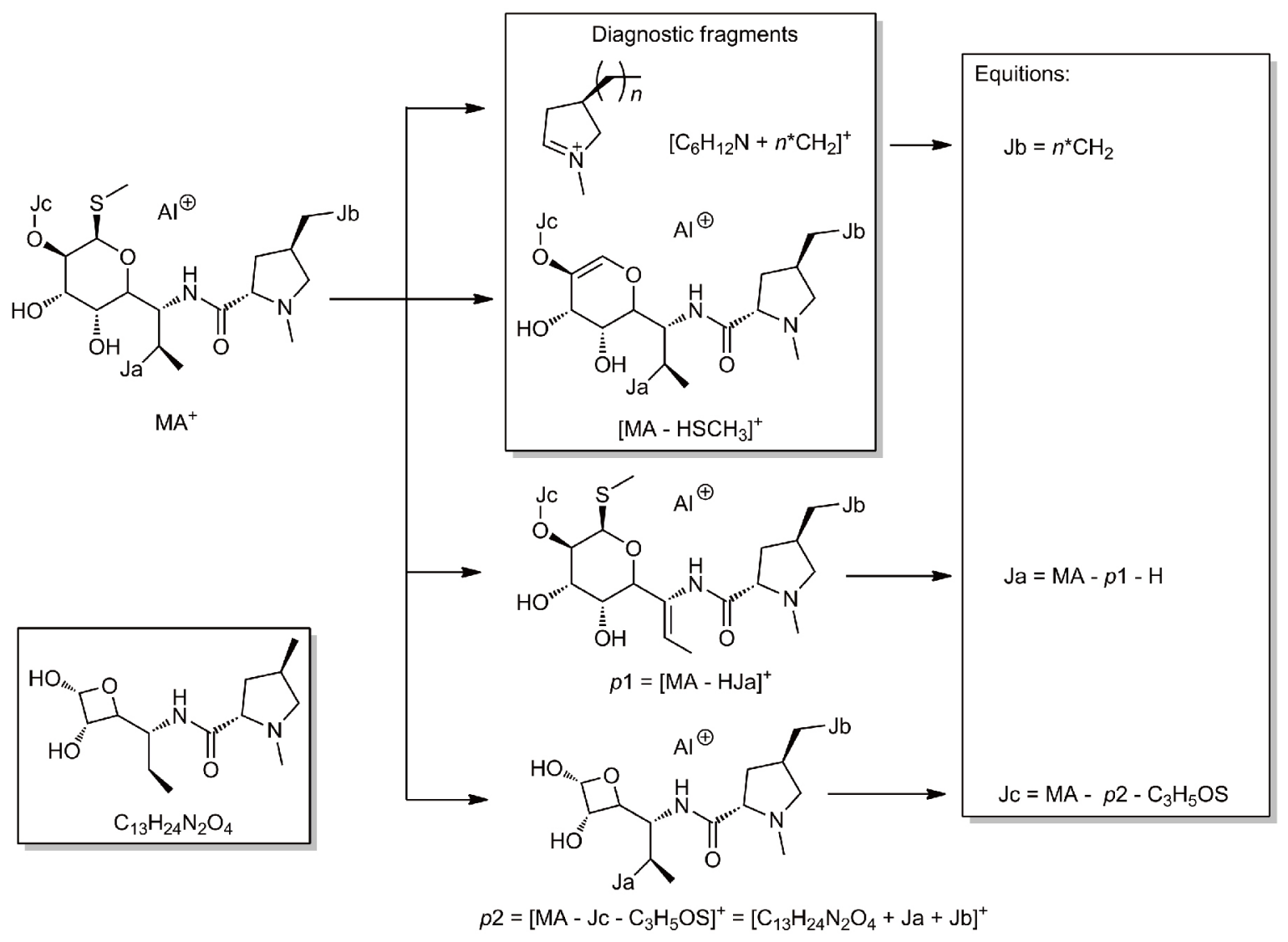

图 4 林可需素类化合物的主要裂解途径及周边基团的计算公式

而

$$
\left[\mathrm{MA}-\mathrm{Jc}-\mathrm{C}_{3} \mathrm{H}_{5} \mathrm{OS}\right]^{+}=\left[\mathrm{C}_{13} \mathrm{H}_{24} \mathrm{~N}_{2} \mathrm{O}_{4}+\mathrm{Ja}+\mathrm{Jb}\right]^{+}
$$

所以此时必然存在一个峰 $\left[\mathrm{MA}-\mathrm{Jc}^{-}-\mathrm{C}_{3} \mathrm{H}_{5} \mathrm{OS}\right]^{+}$使下 列等式成立:

$$
\begin{aligned}
& {\left[\mathrm{MA}-\mathrm{Jc}-\mathrm{C}_{3} \mathrm{H}_{5} \mathrm{OS}\right]^{+}} \\
& =\left[\mathrm{C}_{13} \mathrm{H}_{24} \mathrm{~N}_{2} \mathrm{O}_{4}+\mathrm{Ja}+\mathrm{Jb}\right]^{+} \\
& =\left[\mathrm{C}_{13} \mathrm{H}_{24} \mathrm{~N}_{2} \mathrm{O}+(\mathrm{MA}-p 1-\mathrm{H})+n * \mathrm{CH}_{2}\right]^{+} \\
& =\left[\mathrm{C}_{13} \mathrm{H}_{24} \mathrm{~N}_{2} \mathrm{O}_{4}+\mathrm{MA}-p 1-\mathrm{H}+n * \mathrm{CH}_{2}\right]^{+}
\end{aligned}
$$

即在变量 $p 1$ 遍历整个串联质谱的质荷比时, 若存 在一个峰满足 $p=\left[\mathrm{C}_{13} \mathrm{H}_{24} \mathrm{~N}_{2} \mathrm{O}_{4}+\mathrm{MA}-p 1-\mathrm{H}+n^{*} \mathrm{CH}_{2}\right]^{+}$, 则 可以认为此峰 $p 1$ 即为 $[\mathrm{MA}-\mathrm{HJa}+\mathrm{H}]^{+}$, 而对应的 $\mathrm{Ja}, \mathrm{Jb}, \mathrm{Jc}$ 即可按照下列公式计算得出:

$$
\begin{gathered}
\mathrm{Ja}=\mathrm{MA}-[\mathrm{MA}-\mathrm{HJa}]^{+}-\mathrm{H}=\mathrm{MA}-p 1-\mathrm{H} \\
\mathrm{Jb}=n * \mathrm{CH}_{2}
\end{gathered}
$$

$$
\begin{aligned}
\mathrm{Jc} & =\mathrm{MA}-\left[\mathrm{MA}-\mathrm{Jc}-\mathrm{C}_{3} \mathrm{H}_{5} \mathrm{OS}\right]^{+}-\mathrm{C}_{3} \mathrm{H}_{5} \mathrm{OS} \\
& =\mathrm{MA}-p-\mathrm{C}_{3} \mathrm{H}_{5} \mathrm{OS}
\end{aligned}
$$

将最后得出的这 3 个公式翻译成解析规则(图S2) 后储存于本地解析规则数据库中.

某待分析林可霉素化合物的MS/MS数据被手工 输入到SmartMass里面, 在正确设置好了极性、加合 离子和母离子的 $m / z$ 值之后, 点击Analyze按钮进行分 析, 得到了 clindamycin (图S3(a)) 的结构, 进一步使用 PubChem进行确认的图见图S3(b, c), 解析代码的预览 见图S3(d).

经过 SmartMass 解析后我们得到了正确的 clindamycin结构, 然而仅通过解析结果无法知道该结构是 已知还是未知结构. 因此对此结构进行了进一步的验 证, SmartMass利用精确质量数/分子量在PubChem数 据库中进行检索, 然后化合物的特征结构(骨架类型) 对所获得的候选结构进行笁选, 最终获得了 49 个候选 结构(图S3), 而所解析出来的结构正在其中, 说明这是 
一个已知化合物. 以上说明 SmartMass 在天然产物去 重复性篎选中具有重要的指导价值.

\section{3 米团花新型二倍半萜化合物的分析}

中国科学院昆明植物所黎胜红课题组 ${ }^{[30]}$ 在唇形 科大型木本植物米团花 (Leucosceptrum canum Smith) 的腺毛中发现了一类骨架新颖的二倍半萜类化合物, 此类二倍半萜类化合物与米团花昆虫防御行为有关. 对此类化合物质谱裂解机理的研究, 有助于快速发现 新的类似化合物, 以及确定此类化合物在植物中不同 部位的分布, 深入理解植物与昆虫之间的相互生存竞 争现象具有重要意义. 我们在研究中发现, 该类化合 物很难质子化, 即使在溶液中加入挥发性酸, 其准分 子离子峰 $\left([\mathrm{M}+\mathrm{H}]^{+}\right)$信号依然较弱, 相反 $[\mathrm{M}+\mathrm{Na}]^{+}$离子和 $[\mathrm{M}+\mathrm{K}]^{+}$离子信号较强. 但在串联质谱条件下, 二倍半 萜类化合物的加合离子均无法产生骨架裂解碎片. 最 终, 我们通过不断尝试发现在溶液中添加锂离子后, 不仅在一级质谱中产生高丰度的 $[\mathrm{M}+\mathrm{Li}]^{+}$离子, 而且 在串联质谱中, 产生了特征性的骨架裂解. 除了产生 $\left[\mathrm{M}+\mathrm{Li}-\mathrm{H}_{2} \mathrm{O}\right]^{+}$信号, 同时发生 $\mathrm{C}$ 环的开裂, 产生该类化
合物特征的离子. 通过两个途径( $(\mathrm{a}$ 和 $\mathrm{b})$ 进行 $\mathrm{C}-\mathrm{O}$ 键的 断裂, 两个途径同时发生, $\mathrm{a}$ 途径为 $\mathrm{C} 4-\mathrm{O}$ 键的断裂, 产 生包含 $\mathrm{D}$ 环侧链的碎片离子; 而 $\mathrm{b}$ 途径为 $\mathrm{C} 14-\mathrm{O}$ 键的断 裂, 然后生成包含分子母核结构的碎片离子 $[264+\mathrm{Li}]^{+}$ 离子或 $[248+\mathrm{Li}]^{+}$离子, 其可能裂解方式见图 5 .

因此类型化合物的 $\mathrm{R} 2$ 基团相对变化较少, 可以通 过串联质谱信号中是否存在含 $\mathrm{R} 2$ 基团的母核碎片来 初步判断是否为此类化合物, 即该类化合物的特征碎 片为a裂解途径的 $m / z 271$ 或 255 . 若在串联质谱中发现 $m / z 255$ 则可判断其 $\mathrm{R} 2$ 为 $-\mathrm{H}$, 出现 $m / z 271$ 离子意味着 其 $\mathrm{R} 2$ 为 $-\mathrm{OH}$. 此外, 通过增加 $\mathrm{b}$ 途径特征碎片离子的判 断可以更准确地判断是否为此类化合物, 即 $\mathrm{R} 2$ 为 $-\mathrm{H}$ 和 $-\mathrm{OH}$ 时判断是否出现 $m / z 273$ 和 289 特征离子. 对于 $\mathrm{R} 1$ 基团, 可以通过出现 $m / z 273$ 和 289 特征离子时母离 子质荷比与特征离子质荷比之差来进行计算, 并在 可能的 R1基团数据库中进行查找. 因此, 我们的解析 规则通过判断是否出现特征碎片 $m / z 255$ 和 273 来确定 $\mathrm{R} 2$ 是否为 $-\mathrm{H}$, 若 $m / z 271$ 和 289 同时出现, 则表明其 $\mathrm{R} 2$ 为 $-\mathrm{OH}$. R1则通过母离子与特征碎片离子的质荷比差 值进行查找. 我们根据此解析策略编写了解析规则 (见

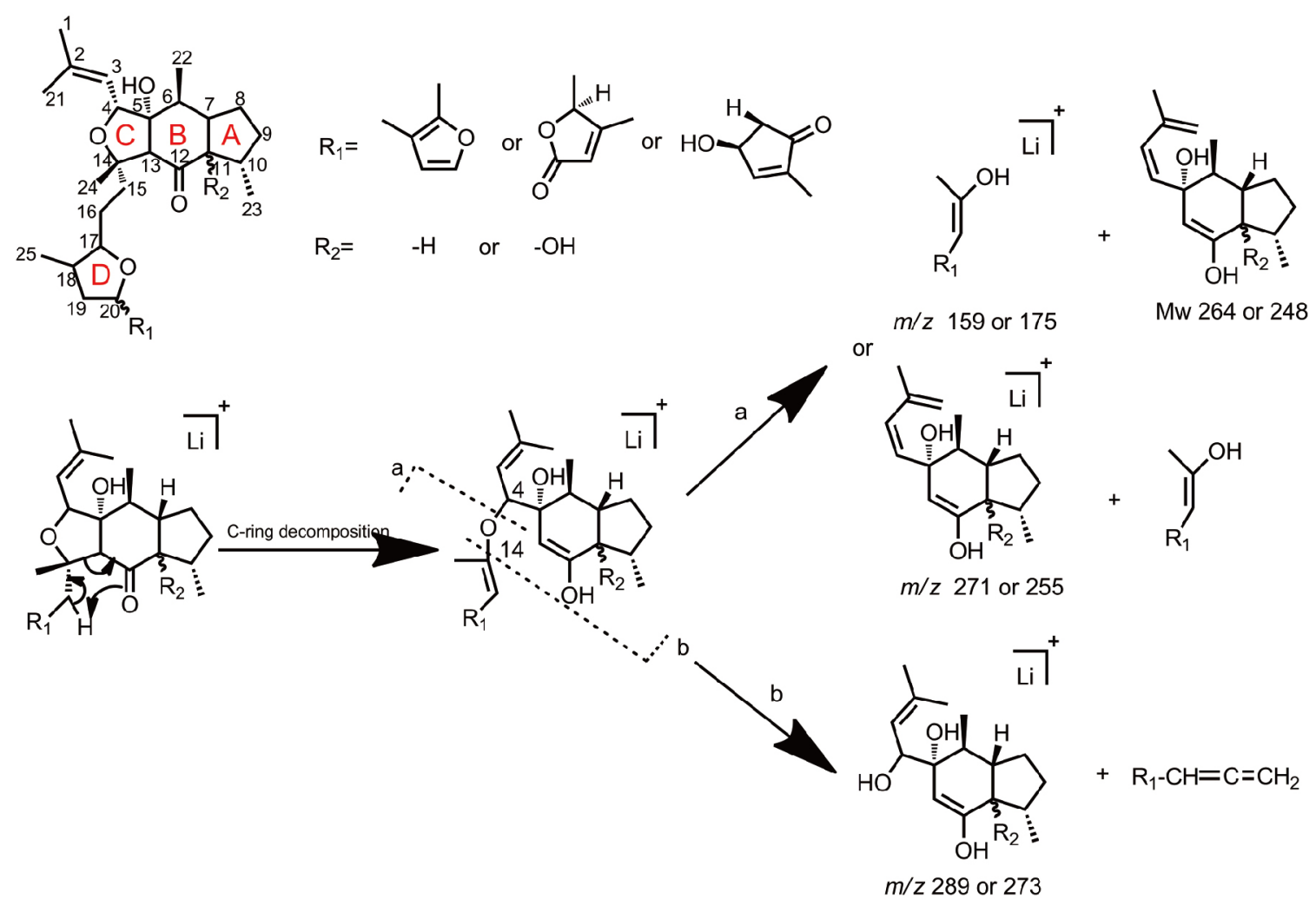

图 5 米团花二倍半萜的结构及质谱裂解途径

1464 
网络版支持信息S2 小节及图S5).

我们对米团花提取物进行液质联用分析, 从中获 取一个未知化合物的 MS/MS (图S6) 数据被手工输入 到SmartMass里面, 在设置好了极性、加合离子和母 离子的 $m / z$ 值之后, 点击Analyze进行分析, 得到了该化 合物可能的结构(图S7).

\section{4 结论}

本课题组开发了基于天然化合物质谱裂解的 SmartMass智能解析软件. MS/MS实验表明, 具有相 同骨架类型的天然化合物的质谱裂解存在一定的特征 裂解规律, 这是SmartMass的解析基础. 在结构解析思 路上看, 该方法要优于谱图匹配的方法. SmartMass依 靠的是质谱裂解规律, 而谱图匹配依靠的是已知化合 物已有的质谱数据. 因此, SmartMass 可以推测属于已 知化合物类型的任何化合物, 包括周边基团变异了的 未知化合物, 而常规技术却不能. 因其非目标性的特 点, SmartMass在天然产物、药物合成、药物代谢及食 品安全等领域均有潜在的应用价值, 通过完善相关解
析数据库, 本技术有望在上述领域获得应用, 提升研究 人员的效率. 首先, 在快速分析鉴定天然产物化合物 成分上有着非常明显的优势, 天然产物大多都属于特 定的骨架类型(黄酮类、皇苷类、生物碱类等), 通过 总结出该类化合物的特征裂解规律并编写解析规则, 就可以快速鉴定分析其中植物中的化合物成分, 包括 已知成分和未知的同类型化合物, 为去重复分离和新 化合物的发现提供有益的参考; 在药物合成中, 会产 生一些具有相同骨架类型的杂质, 通过该程序可以很 容易辨认并推测杂质分子的结构; 在药物代谢中, 多 数药物分子经过体内的代谢生成了一系列具有相同 骨架类型的化合物, 通过已有裂解规律, 可以很快知道 生成了何种产物; 在食品安全中, 利用SmartMass可以 很快发现一些经过修饰或改造了的违禁添加剂; 在毒 品检测中, 亦可通过质谱很快地鉴定出可能的被修饰 过了的毒品、药物. 因此, SmartMass 可以提高分析工 作者的效率. 我们将此软件托管在Google Code上, 其 对应的项目链接为 http://code.google.com $/ \mathrm{p} / \mathrm{smartmass,}$ 欢迎大家参与项目或者报告程序Bug和提出建议.

\section{支持信息}

本文的支持信息见网络版chem.scichina.com. 支持信息为作者提供的原始数据，作者对其学术质量和内容负责.

\section{参考文献}

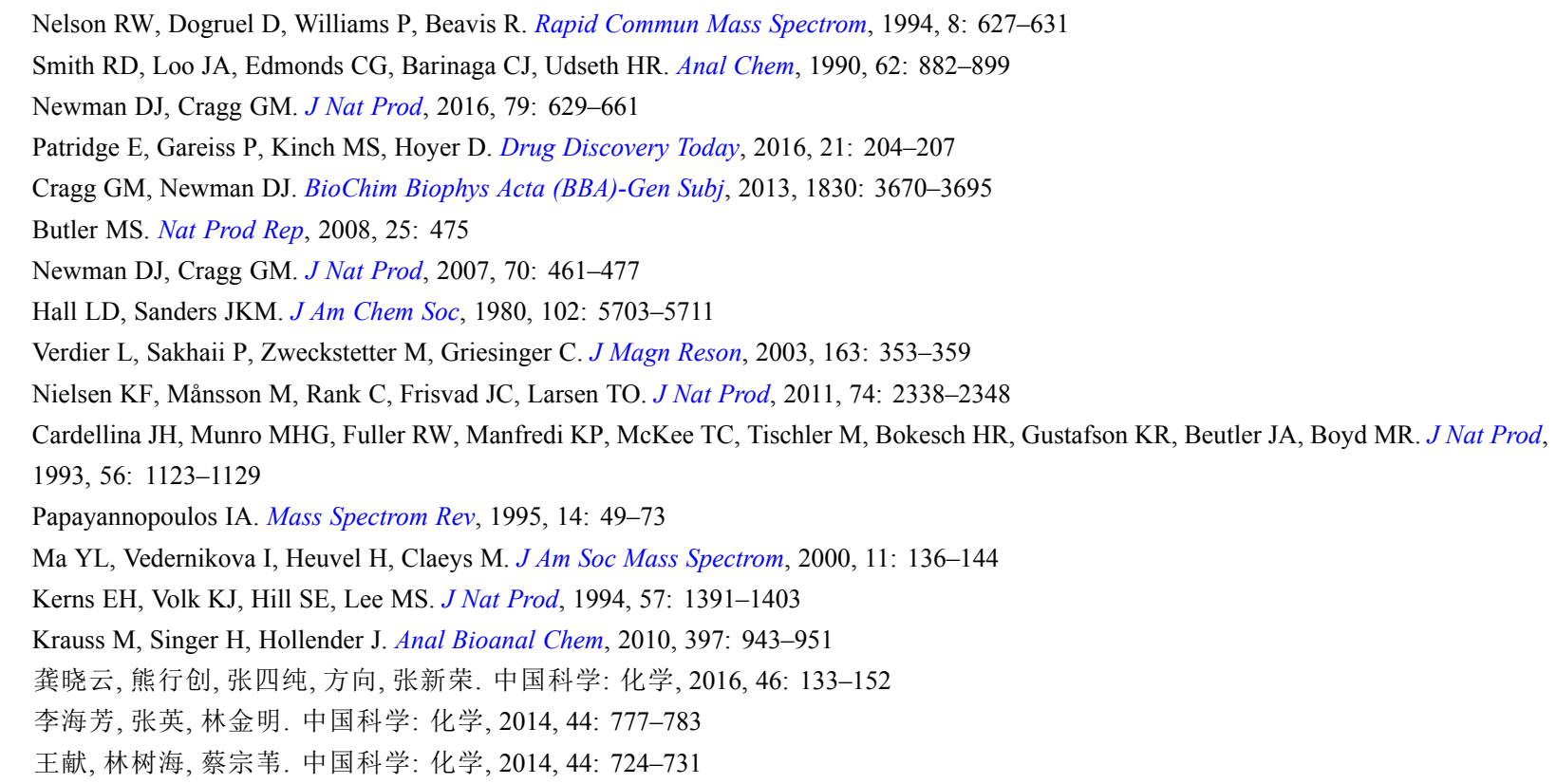


19 Lindsay RK, Buchanan BG, Feigenbaum EA, Lederberg J. Applications of Artificial Intelligence for Organic Chemistry: the DENDRAL Project. New York: McGraw-Hill Book Company, 1980

20 Baričič P, Mackov M. J Mol Graphics, 1995, 13: 184-189

21 Hart PE, Duda RO, Einaudi MT. Math Geol, 1978, 10: 589-610

22 High Chem Mass Frontier 7.0 SR1, 2012. Available at: http://www.highchem.com

23 ACD/MS Fragmenter, 2012. Available at: http://www.acdlabs.com

24 Mascot, 2012. Available at: http://www.matrixscience.com

25 Xia B, Liu X, Gu YC, Zhang ZH, Wang HY, Ding LS, Zhou Y. J Am Soc Mass Spectrom, 2013, 24: 789-793

Xia B, Tai ZF, Gu YC, Li BJ, Ding LS, Zhou Y. J Comput Chem, 2011, 32: 2942-2948

Wang Y, Xiao J, Suzek TO, Zhang J, Wang J, Bryant SH. Nucleic Acids Res, 2009, 37: W623-W633

Bryant S. PubChem: an information resource linking chemistry and biology. In: Abstracts of Papers of the American Chemical Society. San Francisco, CA, 2006. 231

29 Yang XW, Yang J, Xu G. J Nat Prod, 2017, 80: 108-113

30 Luo SH, Luo Q, Niu XM, Xie MJ, Zhao X, Schneider B, Gershenzon J, Li SH. Angew Chem Int Ed, 2010, 49 : 4471

\title{
Intelligent interpretation of mass spectra of natural products
}

\author{
Bing Xia, Fengwei Ma, Lisheng Ding, Yan Zhou* \\ Chengdu Institute of Biology, Chinese Academy of Sciences, Chengdu 610041, China \\ *Corresponding author (email: zhouyan@cib.ac.cn)
}

\begin{abstract}
Tandem mass spectrometry (MS/MS) plays an important role in the screening and structural characterization of natural products. In addition to general fragmentations, chemicals sharing a same particular structure often produce a common characteristic fragmentation pattern under MS/MS experiments which represent a specific characteristic substructure. Therefore, the class of unknown natural products can be predicted by the diagnostic fragment ions based on MS/MS. The peripheral groups of the chemicals within a class could be inferred by general fragmentation pattern. Based on above mentioned concepts, SmartMass, a software program was developed and implemented for intelligent interpretation of natural products.
\end{abstract}

Keywords: natural products, mass spectrometry, characteristic fragmentation, intelligent interpretation of mass spectra

doi: $10.1360 / \mathrm{N} 032017-00115$ 